\title{
Application of Time-Lapse Video Microscopy in Studying Lung Cancer Cellular Cytoskeletal Functions under Targeted Kinase Inhibitor Novel Therapy.
}

\author{
Patrick C. Ma*\#, Runlei Du*, Zhe Tang* \\ * Division of Hematology/Oncology, Department of Medicine, Case Western Reserve University. \\ \# University Hospitals Case Medical Center, Case Comprehensive Cancer Center, Cleveland, OH.
}

Lung cancer has a high incidence and is the leading cause of cancer death in the United States, resulting in 161,840 deaths in 2008. Receptor tyrosine kinases (RTKs) play key roles in lung cancer tumorigenesis and progression (1). Non-small cell lung cancer accounts for majority cases of lung cancer. Traditional cytotoxic chemotherapy has reached a plateau in the treatment of advanced lung cancer. Progress has been made recently using small molecule tyrosine kinase inhibitors (TKIs) gefitinib and erlotinib, targeting epidermal growth factor receptor (EGFR/ERBB1/HER1) (2). EGFR kinase domain mutations have been identified to be predictive of response to EGFR TKIs. However, majority of unselected lung cancer patients are still primary non-responders. Initial responders with mutant-EGFR invariably develop secondary resistance and soon succumb to the disease. At least half of the acquired resistance is mediated by the "gatekeeper" mutation T790MEGFR $(3,4)$. T790M was also found in the TKI-naïve NCI-H1975 cell line, in combination with L858R. Currently, there are still no Federal Drug Administration (FDA)-approved clinical inhibitors that can overcome T790M-mediated EGFR-TKI resistance yet. The RTK MET also play important roles in human cancers $(5,6)$ and has been validated as an attractive therapeutic target in lung cancer $(5,7,8)$. MET may have a role in the acquired resistance to EGFR TKI $(9,10)$. Dysregulation of the MET-HGF/SF (hepatocyte growth factor/scatter factor) signaling axis upregulates diverse tumor cell functions including cell survival, cell scattering and motility, epithelial-mesenchymal transition, invasion and metastasis. We showed that MET is highly expressed in lung cancer, often concomitantly with EGFR. The erlotinib-resistant lung cancer cell line NCI-H1975 (expressing wild type MET and T790M-EGFR) was used to test for the effect of MET inhibition. Small molecule inhibitors such as SU11274 (8) and PHA665752 (11) targeting MET have been developed to test for therapeutic inhibition in lung cancer. In order to study combined MET-EGFR targeted inhibition to optimize lung cancer treatment in the erlotinib-resistant H1975 cells, we have applied in vitro timelapsed video microscopy (TLVM) and in vivo luciferase-bioluminescence(BLI) imaging platform, monitoring the in vitro cytoskeletal functions (cell motility and migration)(Fig. 1) and in vivo tumor xenograft growth (Fig. 2) respectively in response to the combined TKIs.

In the TLVM and in vivo BLI studies, dual SU11274-erlotinib concurrent treatment effectively inhibited H1975 cells with enhanced abrogation of cytoskeletal functions and complete regression of the xenograft growth. For TLVM assay, H1975 cells were cultured in either serumstarved or $10 \%$ FBS media, and placed into a temperature-controlled chamber at $37^{\circ} \mathrm{C}$ in an atmosphere of $5 \% \mathrm{CO}_{2}$. The cells were examined by TLVM using a Leica $6000 \mathrm{~B}$ inverted microscope, Pecon incubation chamber and Retiga EXI 12 bit camera (Q imaging, Vancouver, B.C.). For cell movement analysis, H1975 cells with/without kinase inhibitor treatment as indicated were monitored under TLVM with digital images digitally recorded for a total of 18 hours, in quadruplicates. Cellular cytoskeletal functions were analyzed using the MetaMorph image analysis software (Universal Imaging), with cell movement trajectories plotted in different highlighted colors. Together, our results suggest that MET-based targeted inhibition using MET inhibitor can be a 
potential treatment strategy for T790M-EGFR mediated erlotinib-resistant NSCLC. Furthermore, optimized inhibition may be further achieved with MET inhibition in combination with EGFR-TKI.

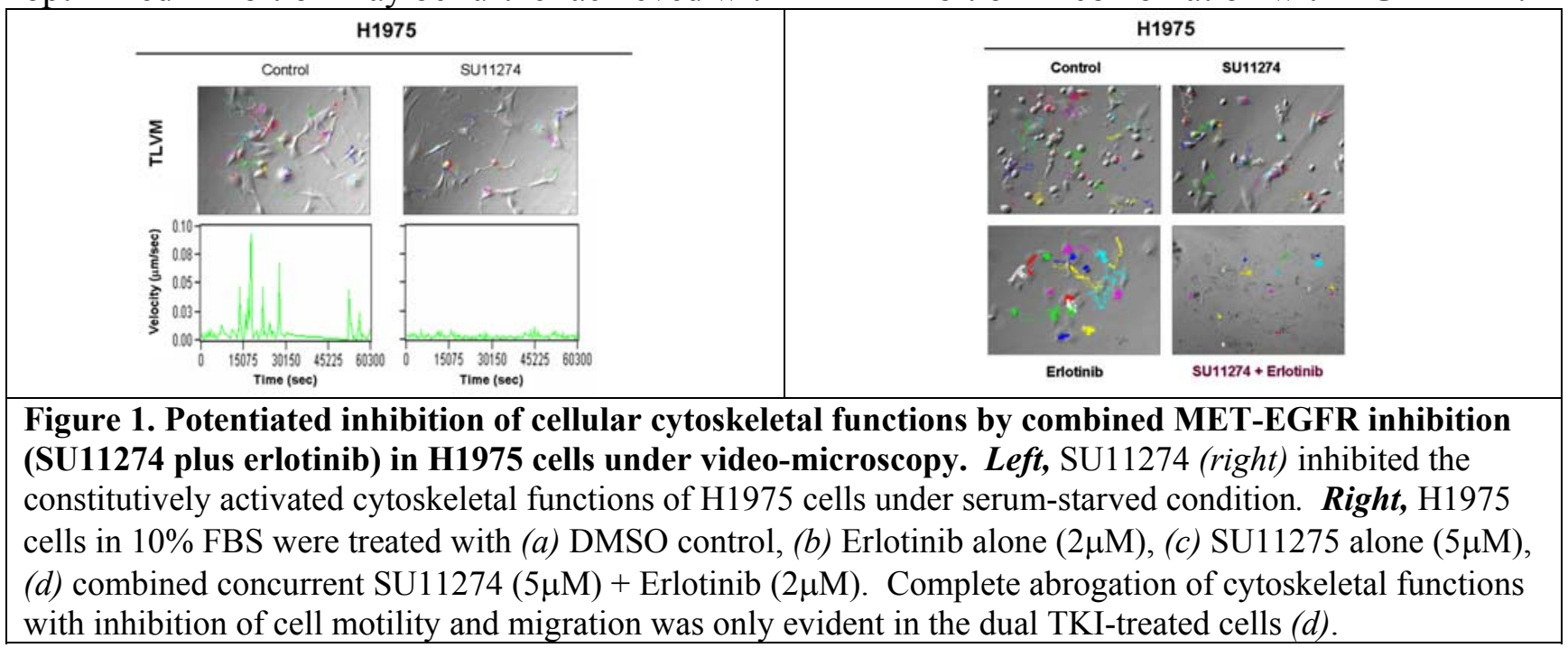
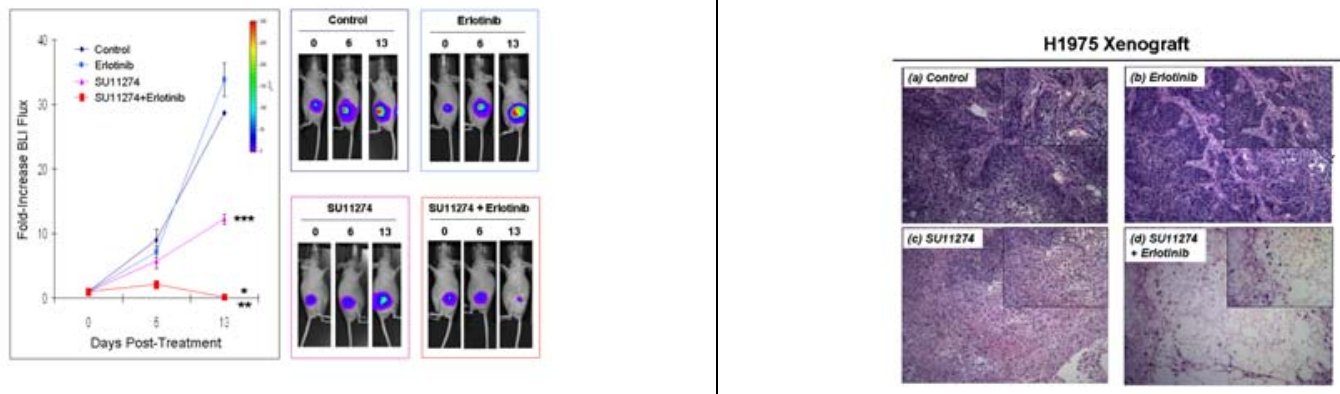

Figure 2. In vivo treatment using SU11274 combined with erlotinib induced cooperative complete regression of EGFR-TKI resistant $\mathrm{H1975}$ tumor xenograft growth. Left, EGFR-TKI-resistant H1975-luc cells established in nude mouse xenograft in vivo were treated as indicated and assayed under BLI.

Right, H \& E staining at 100x mag (and 200x, inset) showed massive tumor necrosis/apoptosis in the combined SU11274 plus erlotinib-treated animals $(d)$.

\section{References}

1. N. W. Choong, P. C. Ma, R. Salgia, Expert Opin Ther Targets 9, 533 (2005).

2. T. J. Lynch et al., Clin Cancer Res 12, 4365s (2006).

3. S. Kobayashi et al., N Engl J Med 352, 786 (2005).

4. W. Pao et al., PLoS Med 2, e73 (2005).

5. P. C. Ma, G. Maulik, J. Christensen, R. Salgia, Cancer Metastasis Rev 22, 309 (2003).

6. C. Birchmeier, et al. Nat Rev Mol Cell Biol 4, 915 (2003).

7. J. G. Christensen, J. Burrows, R. Salgia, Cancer Lett 225, 1 (2005).

8. P. C. Ma et al., Cancer Res 65, 1479 (2005).

9. J. A. Engelman et al., Science 316, 1039 (2007).

10. J. Bean et al., Proc Natl Acad Sci U S A 104, 20932 (2007).

11. P. C. Ma, E. Schaefer, J. G. Christensen, R. Salgia, Clin Cancer Res 11, 2312 (2005).

12. This work was supported by NIH/NCI-K08 (4K08CA102545-04), the Case Comprehensive Cancer Center (Confocal Microscopy Core and Animal Imaging Core Facilities) of CWRU/UH Case Medical Center (P30 CA43703-12). 